\title{
High-spin states and a new band based on the isomeric state in ${ }^{152} \mathrm{Nd}$
}

\author{
E.Y. Yeoh ${ }^{1, a}$, S.J. Zhu ${ }^{1,2, b}$, J.H. Hamilton ${ }^{2}$, A.V. Ramayya ${ }^{2}$, Y.C. Yang ${ }^{3}$, Y. Sun ${ }^{3}$, J.K. Hwang ${ }^{2}$, S.H. Liu ${ }^{2}$, \\ J.G. Wang ${ }^{1}$, Y.X. Luo $^{2,4}$, J.O. Rasmussen ${ }^{4}$, I.Y. Lee ${ }^{4}$, H.B. Ding ${ }^{1}$, K. Li ${ }^{2}$, L. Gu ${ }^{1}$, Q. Xu ${ }^{1}$, Z.G. Xiao ${ }^{1}$, and W.C. Ma ${ }^{5}$ \\ 1 Department of Physics, Tsinghua University, Beijing 100084, PRC \\ 2 Department of Physics, Vanderbilt University, Nashville, TN 37235, USA \\ 3 Department of Physcis, Shanghai Jiao Tong University, Shanghai 200240, PRC \\ 4 Lawrence Berkeley National Laboratory, Berkeley, CA 94720, USA \\ 5 Department of Physics, Mississippi State University, Mississippi State, MS 39762, USA
}

Received: 15 January 2010 / Revised: 22 April 2010

Published online: 6 July 2010

(C) The Author(s) 2010. This article is published with open access at Springerlink.com

Communicated by C. Signorini

\begin{abstract}
High-spin states of the neutron-rich ${ }^{152} \mathrm{Nd}$ nucleus have been reinvestigated by measuring the prompt $\gamma$-rays in the spontaneous fission of ${ }^{252} \mathrm{Cf}$. The ground-state band and a side negative-parity band have been updated. A new band based on the $2243.7 \mathrm{keV}$ isomeric state has been identified. The half-life for the isomeric state has been measured to be $63(7) \mathrm{ns}$. The projected shell model is employed to study the band structure of this nucleus. The results show that the calculated levels of the bands are in good agreement with the experimental ones, and the isomeric state and the negative-parity band are based on the proton $\pi 5 / 2^{-}[532] \otimes \pi 9 / 2^{+}[404]$ and neutron $\nu 3 / 2^{-}[521] \otimes \nu 5 / 2^{+}[642]$ two-quasiparticles configurations, respectively.
\end{abstract}

The neutron-rich nuclei around the $A \sim 150$ region show transitional character from spherical to prolate quadrupole deformation and from octupole vibrational excitations to static octupole deformation [1-5]. ${ }^{152} \mathrm{Nd}$ is located in the deformed region and has a well-deformed shape. Previously, the level structure of ${ }^{152} \mathrm{Nd}$ has been studied by using various experimental methods [6-12]. The ground-state band and a negative-parity band based on a $2^{-}$state have been identified. In addition, the identification of the $K$ isomeric states and the bands built on the isomers is a very important topic in this region. In a recent publication, two-quasiparticle (2-qp) isomeric states and bands based on the isomers have been reported in ${ }^{154,156} \mathrm{Nd}$ and ${ }^{156,158,160} \mathrm{Sm}[13]$. For ${ }^{152} \mathrm{Nd}$, an isomeric state was also reported in ref. [11], but no band structure based on that isomer was observed. In order to understand the systematic structural characteristics and to search for the isomeric band, we reinvestigated the high-spin states of ${ }^{152} \mathrm{Nd}$. Here we report our new result.

To study high-spin states of ${ }^{152} \mathrm{Nd}$, the $\gamma$-rays in the spontaneous fission of ${ }^{252} \mathrm{Cf}$ were measured. These $\gamma$-rays include prompt $\gamma$-rays emitted after their formation in spontaneous fission and delayed $\gamma$-rays from $\beta$-decay. The

\footnotetext{
a e-mail: yeoheingyee@gmail.com

b e-mail: zhushj@mail.tsinghua.edu.cn
}

experiment was carried out at the Lawrence Berkeley National Laboratory. The $\gamma-\gamma-\gamma$ coincidence studies were performed with the Gammasphere detector array consisting of 102 Compton-suppressed Ge detectors. The $\gamma-\gamma-\gamma$ and higher-fold coincidence events were triggered by any $\gamma$ ray. The triple- $\gamma$ coincidence method can strongly enhance the prompt $\gamma$-transitions in only that isotope and reduce the delayed $\gamma$-transitions from $\beta$-decay. A ${ }^{252} \mathrm{Cf}$ source of strength $\sim 60 \mu \mathrm{Ci}$ was placed at the center of the Gammasphere. A three-dimensional histogram of $5.7 \times 10^{11}$ coincidence events was constructed. The relative intensities were obtained by calibrating the Gammasphere with ${ }^{133} \mathrm{Ba}$ and ${ }^{152} \mathrm{Eu}$ standard sources. The data have much higher statistics than that in ref. [12]. The coincidence data were analyzed with the Radware software package [14]. More details about the experimental method can be found in refs. $[1,15]$.

By carefully examining the coincidence relationships and $\gamma$-ray relative transition intensities, the level scheme of ${ }^{152} \mathrm{Nd}$ obtained in the present work is shown in fig. 1 . Three collective bands are observed. The new transitions have been marked with the asterisks $(*)$ and the relative intensities of transitions, which are normalized to that of the $164.3 \mathrm{keV}$ transition, are given in parentheses as shown in fig. 1 . In band (3), because the $2243.7 \mathrm{keV}$ level is isomeric (see discussion below), the relative intensities for 
(1)

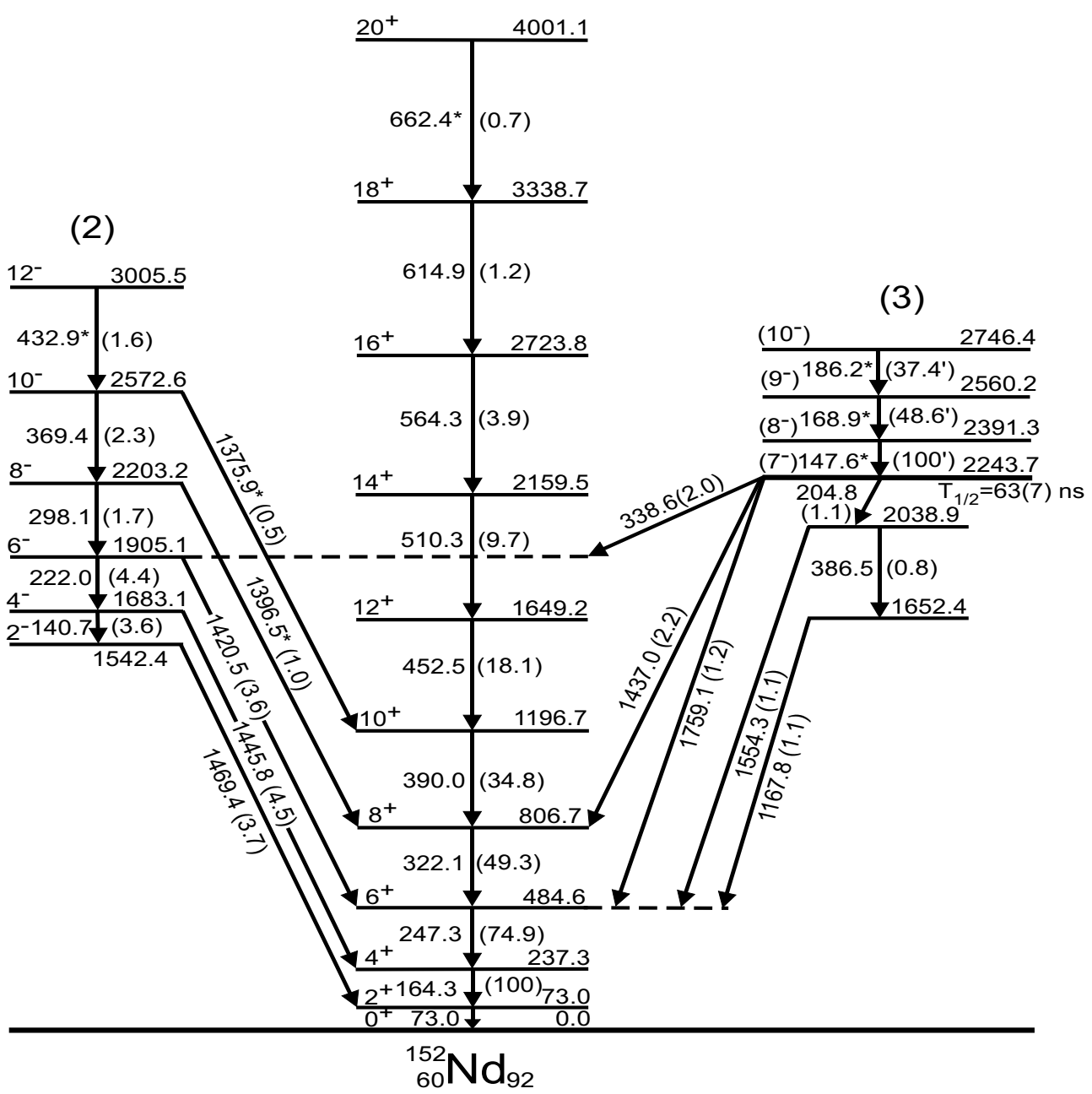

Fig. 1. Level scheme of ${ }^{152} \mathrm{Nd}$ obtained in the present work. Energies are in $\mathrm{keV}$.

the $\gamma$-transitions inside this band cannot be normalized to that of the $164.3 \mathrm{keV}$ transition. So in band (3), for the 168.9 and $186.2 \mathrm{keV}$ transitions, they are only normalized to that of the $147.6 \mathrm{keV}$ one.

The ground-state band (1) of ${ }^{152} \mathrm{Nd}$ was identified up to $18^{+}[10]$, and tentatively expanded to $20^{+}[12]$. In the present work, we confirmed the result up to $18^{+}$. Above that, we add a $662.4 \mathrm{keV} \gamma$-transition $\left(20^{+} \longrightarrow 18^{+}\right)$to band (1) instead of the uncertain $669.3 \mathrm{keV}$ one assigned in ref. [12]. Band (2) based on the $1542.4 \mathrm{keV}$ level was established up to $12^{-}$in ref. [12], but a $435.4 \mathrm{keV}\left(12^{-} \longrightarrow 10^{-}\right)$ transition was also uncertain in ref. [12]. We confirmed this band up to $10^{-}$. Above that, we add a $432.9 \mathrm{keV}$ transition $\left(12^{-} \longrightarrow 10^{-}\right)$to this band instead of the uncertain $435.4 \mathrm{keV}$ one reported in ref. [12]. Band (3) is newly discovered in the present work. The band head level at $2243.7 \mathrm{keV}$ has been identified as an isomeric state with half-life 88(18) ns, along with some de-excited transitions from this isomeric state to the ground-state band [11] Above the isomeric state, three new levels at 2391.3, 2560.2 and $2746.4 \mathrm{keV}$, along with three new $\gamma$-transitions of $147.6,168.9$ and $186.2 \mathrm{keV}$ have been identified in our work. According to the energy spacings inside this band and the systematical comparison with the isomer bands in the neighboring nuclei, we assign the transitions inside band (3) as $M 1$-transitions. Besides, two new side transitions of 1396.5 and $1375.9 \mathrm{keV}$ between bands (2) and (1) are also observed. As examples, fig. 2 shows three coincidence $\gamma$-ray spectra in ${ }^{152} \mathrm{Nd}$. In fig. 2 (a), by double gating on 322.1 and $614.9 \mathrm{keV} \gamma$-transitions, one can see all the $\gamma$-peaks in band (1), except for the gating peaks of 322.1 and $614.9 \mathrm{keV}$. In fig. 2(b), by double gating on 322.1 and $1437.0 \mathrm{keV} \gamma$-transitions, three new transitions of 147.6 , 168.9 and $186.2 \mathrm{keV}$ in band (3) as well as the 73.0, 164.3 and $247.3 \mathrm{keV}$ transitions in band (1) can be seen, and some $\gamma$-transitions of the partner nuclei, such as, $144.9 \mathrm{keV}$ in ${ }^{98} \mathrm{Sr}(2 \mathrm{n})$ and $204.2 \mathrm{keV}$ in ${ }^{95} \mathrm{Sr}(5 \mathrm{n})$ (the numbers in parentheses here indicate the number of neutrons emitted after fission) can also be seen. Figure 2(c) is obtained by double gating on 298.1 and $1420.5 \mathrm{keV} \gamma$-transitions. From this spectrum, in addition to the transitions of 73.0, 164.3 and $247.3 \mathrm{keV}$ in band (1), and $369.4 \mathrm{keV}$ in band (2), a new transition of $432.9 \mathrm{keV}$ in band (2) can also be seen. In these spectra, some peaks which are not labeled belong 


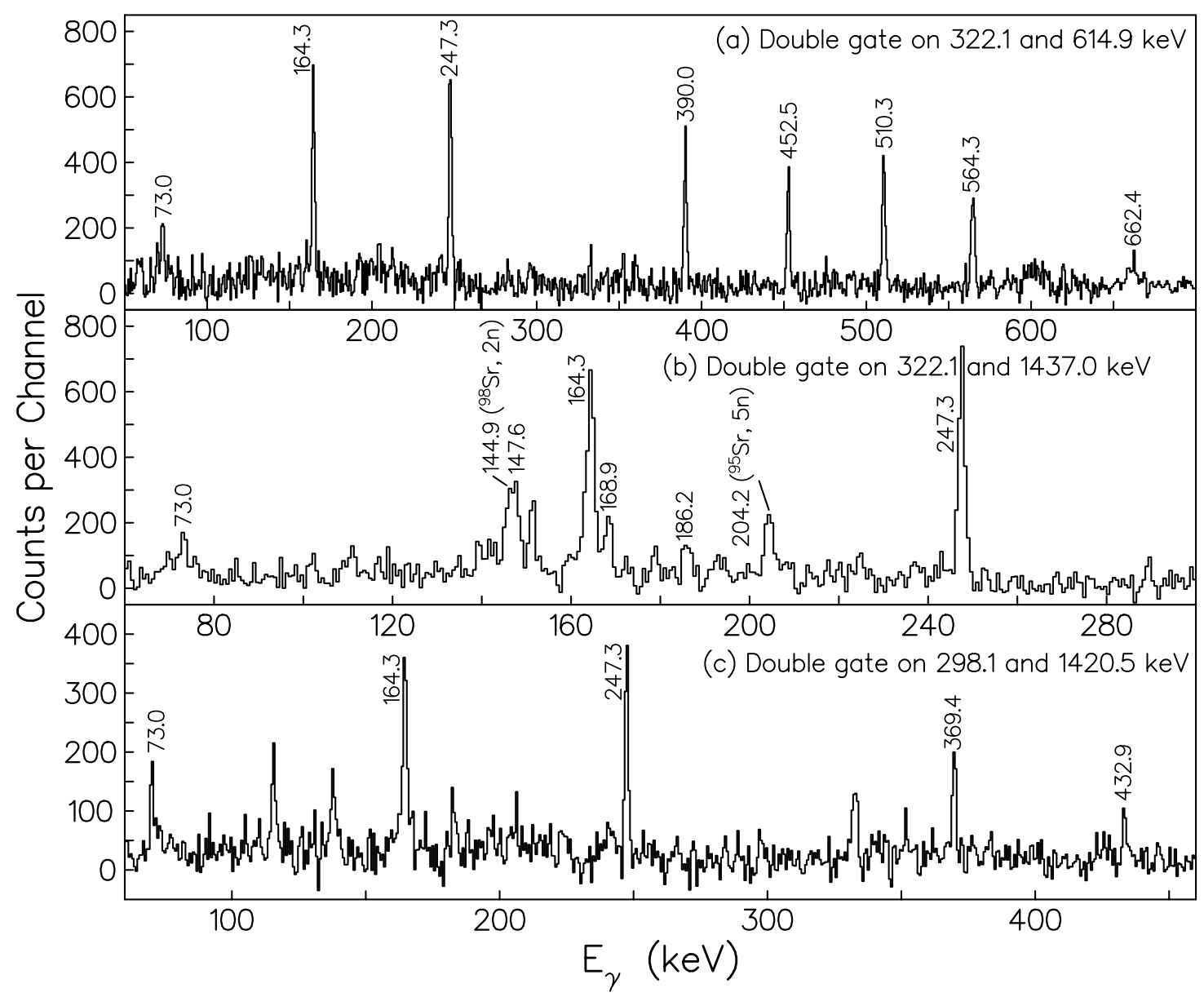

Fig. 2. Portion of $\gamma$-ray spectra by (a) double gating on 322.1 and $614.9 \mathrm{keV}$, (b) double gating on 322.1 and $1437.0 \mathrm{keV}$, and (c) double gating on 298.1 and $1420.5 \mathrm{keV}$, in ${ }^{152} \mathrm{Nd}$.

to coincidence background peaks from other fission fragments. We excluded them from ${ }^{152} \mathrm{Nd}$.

In order to confirm the isomeric state at $2243.7 \mathrm{keV}$ in ${ }^{152} \mathrm{Nd}$ reported in ref. [11], we have carried out the nanosecond half-life analysis from our data by using the delay coincidence matrix method in refs. [16,17]. The measured half-life for this isomeric level is $63(7) \mathrm{ns}$. This result confirms the isomeric state, but it is smaller than the result in ref. [11].

The character of the ground bands in the Nd isotopes has been discussed in refs. [6-8,10,11]. The ratios of $E\left(4_{1}^{+}\right) / E\left(2_{1}^{+}\right)$are 2.49 for ${ }^{148} \mathrm{Nd}, 2.93$ for ${ }^{150} \mathrm{Nd}, 3.25$ for ${ }^{152} \mathrm{Nd}$ and 3.26 for ${ }^{154} \mathrm{Nd}$. These values indicate that these $\mathrm{Nd}$ nuclei are located in a transitional region from spherical to deformed nuclei, and the ${ }^{152} \mathrm{Nd}$ nucleus has a well-deformed shape. The experimental lifetime of the $2^{+}$ state in band (1) for ${ }^{152} \mathrm{Nd}$ indicates that the deformation parameter $\beta_{2}$ is $0.33[7,8]$. The potential energy surface calculations also showed that ${ }^{152,154,156} \mathrm{Nd}$ have large prolate deformation with $\beta \sim 0.35$ [11]. Band (2) in ${ }^{152} \mathrm{Nd}$ has been assigned as a negative-parity band based on a $2^{-}$state $[8,9,12]$. According to the lifetime measurement, this band was suggested as a $K^{\pi}=2^{-}$two-quasiparticle band with $\nu 3 / 2^{-}[532] \otimes \nu 1 / 2^{+}[660]$ configuration [9]. The excitation energy of the band head energy at or above the paring energy gap indicates that this assignment is possible [9]. A similar band structure has also been identified in ${ }^{154} \mathrm{Nd}[12]$. However, they were suggested as octupole vibrational bands [12]. Therefore, the structure of this negative-parity band still need to be examined.

The most interesting finding of the present study is the observed collective band (3) built on the isomeric state in ${ }^{152} \mathrm{Nd}$. This kind of band structures has been identified in neighboring nuclei, ${ }^{154,156} \mathrm{Nd}$, and ${ }^{156,158} \mathrm{Sm}$ in a recent paper [13]. They are based on a $4^{-}$state in ${ }^{154} \mathrm{Nd}$, and $5^{-}$state in ${ }^{156} \mathrm{Nd}$ and ${ }^{156,158} \mathrm{Sm}$, respectively [13]. The quasiparticle rotor model (QPRM) calculations indicated that the $4^{-}$isomeric state has a $\nu 5 / 2^{+}[642] \otimes$ $\nu 3 / 2^{-}[521]$ configuration, and all the $5^{-}$isomers have the $\nu 5 / 2^{+}[642] \otimes \nu 5 / 2^{-}[523]$ configuration. So all these bands built on the isomeric states belong to the neutron 2-qp bands, and these isomers are $K$ isomers. However, examining the decay of the isomeric state at $2243.7 \mathrm{keV}$ of band (3) in ${ }^{152} \mathrm{Nd}$, its spin should be different from those of ${ }^{154,156} \mathrm{Nd}$, and ${ }^{156,158} \mathrm{Sm}$, that is, neither 4 nor 5 . With the transitions observed to only the $6^{+}, 8^{+}$, and $6^{-}$levels, the possible spins and parities $\left(I^{\pi}\right)$ allowed for dipole or $E 2$ radiation of the isomer in ${ }^{152} \mathrm{Nd}$ are $6^{+}, 7^{+}$or $7^{-}$. 


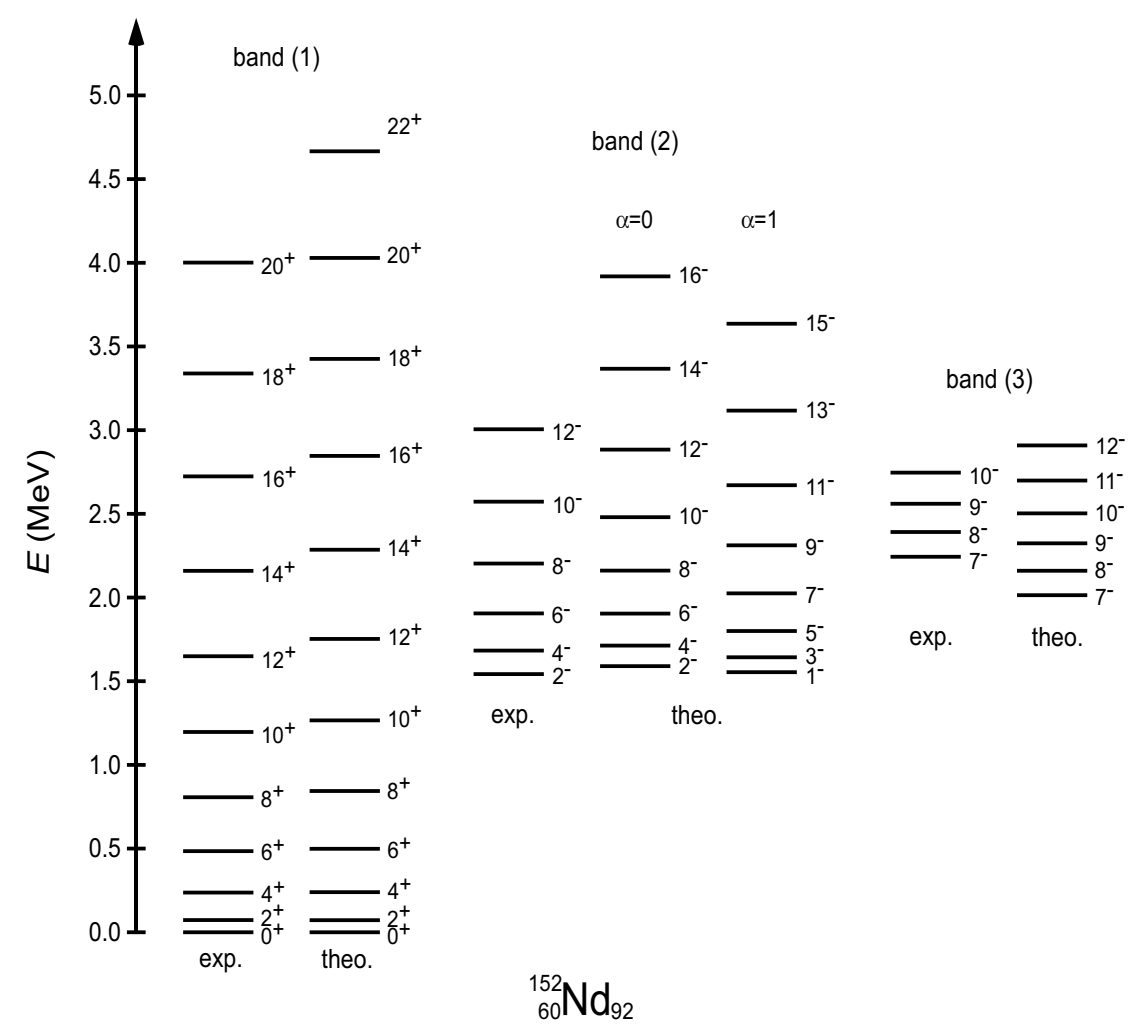

Fig. 3. Comparison of calculated energy levels with experimental data.

We tentatively assigned the $I^{\pi}$ as $7^{-}$. This assignment is supported by the following calculation.

To obtain further insight into the new data, calculations for ${ }^{152} \mathrm{Nd}$ were performed by using the projected shell model (PSM) [18], which has been proven to be successful in high-spin structure studies. The PSM uses the deformed Nilsson single-particle states [19] to start with. Pairing correlations are incorporated into the Nilsson states by the BCS calculation. The consequence of the Nilsson-BCS calculations is to define a set of quasiparticle (qp) states with respect to the qp vacuum $|0\rangle$. One then constructs the shell model bases by building multi-qp states from those Nilsson orbitals that lie close to the Fermi levels. The broken rotational symmetry in the multi-qp states is recovered by exact angular-momentum projection [20] to form a shell model basis in the laboratory frame. Finally, a two-body Hamiltonian is diagonalized in the projected space.

The PSM wave function is a superposition of projected multi-qp states that span the shell model space:

$$
\left|\psi_{M}^{I}\right\rangle=\sum_{\kappa} f_{\kappa}^{I} \hat{P}_{M K_{\kappa}}^{I}\left|\phi_{\kappa}\right\rangle
$$

In eq. (1), $\kappa$ labels the basis states and $f_{\kappa}^{I}$ are determined by the configuration mixing implemented by diagonalization. $\hat{P}_{M K_{\kappa}}^{I}$ is the angular-momentum projection operator [20] which projects an intrinsic configuration $\left|\phi_{\kappa}\right\rangle$ onto states with good angular momentum. As the valence space, particles in three major shells $(N=3,4,5$ for protons and 4, 5, 6 for neutrons) are activated. $\left|\phi_{\kappa}\right\rangle$ consists of 0-, 2- and 4-qp states:

$$
\left|\phi_{\kappa}\right\rangle=\left\{|0\rangle, \alpha_{n_{i}}^{\dagger} \alpha_{n_{j}}^{\dagger}|0\rangle, \alpha_{p_{k}}^{\dagger} \alpha_{p_{l}}^{\dagger}|0\rangle, \alpha_{n_{i}}^{\dagger} \alpha_{n_{j}}^{\dagger} \alpha_{p_{k}}^{\dagger} \alpha_{p_{l}}^{\dagger}|0\rangle\right\}
$$

where $\alpha^{\dagger}$ is the creation operator for a qp and the index $n(p)$ denotes neutron (proton) Nilsson quantum numbers which run over the orbitals close to the Fermi levels.

The PSM Hamiltonian has the form

$$
\hat{H}=\hat{H}_{0}-\frac{1}{2} \chi \sum_{\mu} \hat{Q}_{\mu}^{\dagger} \hat{Q}_{\mu}-G_{M} \hat{P}^{\dagger} \hat{P}-G_{Q} \sum_{\mu} \hat{P}_{\mu}^{\dagger} \hat{P}_{\mu},
$$

where $\hat{H}_{0}$ is the spherical single-particle Hamiltonian which contains a proper spin-orbit force. The monopole pairing strength $G_{M}$ is taken to be $G_{M}=[20.12 \mp$ $13.13(N-Z) / A] / A$ with "-" for neutrons and "+" for protons, respectively. The quadrupole pairing strength $G_{Q}$ is assumed to be proportional to $G_{M}$, the proportionality constant being fixed to 0.18 in the present work. These values are the same as employed in earlier PSM works (see, for example, ref. [21]). Finally, the quadrupolequadrupole interaction strength $\chi$ is determined by the self-consistent relation associated with deformation [18]. As we shall demonstrate below, the use of $\varepsilon_{2}=0.306$ and $\varepsilon_{4}=-0.042$ gives the best description for all the experimentally determined states in ${ }^{152} \mathrm{Nd}$.

The calculated level scheme of ${ }^{152} \mathrm{Nd}$ is compared with the experimental one in fig. 3. Good agreement is found for band (1), the yrast band. The calculation indicates that band (2) originates from the neutron 2-qp 
state $\nu 3 / 2^{-}[521] \otimes \nu 5 / 2^{+}[642]$ with $K^{\pi}=1^{-}$. This assignment disagrees with the early assignment [9]. The suggested configuration in ref. [9] $\left(\nu 3 / 2^{-}[532] \otimes \nu 1 / 2^{+}[660]\right)$ was based on an estimation through possible 2-qp combinations without the support of theoretical calculations that could reproduce the observed levels. The experimentally observed band (2) corresponds to the $\alpha=0$ signature partner which starts from a $2^{-}$state, and another $\alpha=1$ signature partner band which starts from a $1^{-}$state is not observed in our work. So the experimentally observed band (2) is also well described.

The discussion emphasis is now given for the new band (3). It is found that there are two possible candidate configurations with $I^{\pi}=7^{-}$. One is the neutron 2-qp state $\nu 3 / 2^{+}[651] \otimes \nu 11 / 2^{-}[505]$ and the other is the proton $2-q p$ state $\pi 5 / 2^{-}[532] \otimes \pi 9 / 2^{+}[404]$. The calculated $7^{-}$band for the neutron configuration is found higher in energy than the proton configuration, and furthermore, it gives very compressed energy spacings $\left(E_{9^{-}}-E_{7^{-}}=0.220 \mathrm{MeV}\right.$ and $\left.E_{10^{-}}-E_{8^{-}}=0.259 \mathrm{MeV}\right)$, in disagreement with the experimental data $\left(E_{9^{-}}-E_{7^{-}}=0.317 \mathrm{MeV}\right.$ and $E_{10^{-}}-E_{8^{-}}=$ $0.355 \mathrm{MeV})$. The calculated level spacings of the $7^{-}$band for the proton configuration $\left(E_{9^{-}}-E_{7^{-}}=0.310 \mathrm{MeV}\right.$ and $\left.E_{10^{-}}-E_{8^{-}}=0.343 \mathrm{MeV}\right)$, as shown in fig. 3, agree with the experimental ones (i.e. the moment of inertia of the band) although the theoretical band head is about $200 \mathrm{keV}$ below the experimental one. We therefore propose that the newly observed band (3) has a structure of a proton 2-qp configuration.

In conclusion, the high-spin states of the neutronrich ${ }^{152} \mathrm{Nd}$ nucleus have been reinvestigated by measuring the prompt $\gamma$-rays in the spontaneous fission of ${ }^{252} \mathrm{Cf}$. The ground-state band (1) and the side negativeparity band (2) have been expanded. A new band based on the $2243.7 \mathrm{keV}$ isomeric state has been identified in this work. The observed level structures are well described by projected shell model calculations. The projected shell model reproduces the experimental levels, and based on the calculation, we suggest that the isomeric state and the negative-parity band (2) originate from the proton $\pi 5 / 2^{-}[532] \otimes \pi 9 / 2^{+}[404]$ and neutron $\nu 3 / 2^{-}[521] \otimes \nu 5 / 2^{+}[642]$ 2-qp configurations, respectively.
The work at Tsinghua University and Shanghai Jiao Tong University was supported by the National Natural Science Foundation of China under Grants Nos. 10775078, 10975082, 10875077, the Major State Basic Research Development Program under Grand No. 2007CB815005, the Special Program of Higher Education Science Foundation under Grant Nos. 20070003149 and 20090073110061. The work at Vanderbilt University, Mississippi State University and Lawrence Berkeley National Laboratory was supported by U. S. Department of Energy under Grant and Contract Nos. DE-FG05-88ER40407, DE-FG02-95ER40939 and DE-AC03-76SF00098.

Open Access This article is distributed under the terms of the Creative Commons Attribution Noncommercial License which permits any noncommercial use, distribution, and reproduction in any medium, provided the original author(s) and source are credited.

\section{References}

1. J.H. Hamilton et al., Prog. Part. Nucl. Phys. 35, 635 (1995).

2. S.J. Zhu et al., Phys. Lett. B 357, 273 (1995).

3. W.R. Phillips et al., Phys. Rev. Lett. 57, 3257 (1986).

4. S.J. Zhu et al., Phys. Rev. C 59, 1316 (1999).

5. Y.J. Chen et al., Phys. Rev. C 73, 054316 (2006).

6. T. Karlewski et al., Z. Phys. A 330, 55 (1998).

7. M. Hellström et al., Phys. Rev. C 43, 1462 (1991).

8. M. Hellström et al., Phys. Rev. C 46, 860 (1992).

9. M. Hellström et al., Phys. Rev. C 47, 545 (1993).

10. S.J. Zhu et al., J. Phys. G: Nucl. Part. Phys. 21, L75 (1995).

11. C. Gautherin et al., Eur. Phys. J. A 1, 391 (1998).

12. X.Q. Zhang et al., Phys. Rev. C 57, 2040 (1998).

13. G.S. Simpson et al., Phys. Rev. C 80, 024304 (2009).

14. D.C. Radford et al., Nucl. Instrum. Methods Phys. Res. A 361, 297 (1995).

15. Y.X. Luo et al., Phys. Rev. C 64, 054306 (2001).

16. J.K. Hwang et al., Phys. Rev. C 73, 044316 (2006).

17. M.L. Li et al., Chin. Phys. Lett. 21, 2147 (2004).

18. K. Hara, Y. Sun, Int. J. Mod. Phys. E 4, 637 (1995).

19. T. Bengtsson, I. Ragnarsson, Nucl. Phys. A 436, 14 (1985).

20. P. Ring, P. Schuck, The Nuclear Many Body Problem (Springer-Verlag, New York, 1980).

21. Y. Sun, J.L. Egido, Nucl. Phys. A 580, 1 (1994). 